\title{
Efecto de la fragmentación del hábitat sobre la calidad de las semillas en Lapageria rosea
}

\author{
Effects of habitat fragmentation on seed quality of Lapageria rosea
}

CAROLINA A. HENRÍQUEZ

Departamento de Ciencias Ecológicas, Facultad de Ciencias, Universidad de Chile, Casilla 653, Santiago, Chile; e-mail: chenriqu@uchile.cl

\begin{abstract}
RESUMEN
La fragmentación del hábitat tiene un efecto negativo sobre la adecuación biológica de las plantas por la reducción en la cantidad de semillas producidas. Sin embargo, la fragmentación del hábitat también podría disminuir la adecuación biológica de las plantas a través de la reducción en la calidad de las semillas producidas, al reducir atributos como la viabilidad, tamaño o su capacidad germinativa. Esto podría ocurrir porque la reducción en los tamaños poblacionales en fragmentos, junto con el aumento en el grado de aislamiento, podrían generar una reducción de la variabilidad genética de las poblaciones e inducir a la expresión de depresión por endocruza. En este trabajo muestro el efecto de la fragmentación del bosque maulino sobre la calidad de las semillas producidas en la especie Lapageria rosea. Calidad es definida aquí como viabilidad, tamaño y capacidad germinativa de las semillas. Colecté semillas desde fragmentos de bosque y desde un bosque continuo, en el bosque maulino de Chile central. En al laboratorio se estimaron los porcentajes de viabilidad, el tamaño y la capacidad germinativa de las semillas colectadas. Los resultados muestran que las semillas producidas en fragmentos de bosque presentan porcentajes de viabilidad similares a las semillas producidas en el bosque continuo, sin embargo la variabilidad en estos porcentajes es mayor en las semillas de los fragmentos. Además, las semillas de fragmentos son de menor tamaño y poseen menor capacidad germinativa que las semillas de bosque continuo. Estas características de las semillas podría determinar que en fragmentos de bosque exista una reducción en el establecimiento de nuevos individuos, pudiendo inducir a un efecto negativo sobre la sobrevivencia de las poblaciones a largo plazo.
\end{abstract}

Palabras clave: fragmentación del hábitat, semillas, Lapageria rosea, Chile central.

\begin{abstract}
Habitat fragmentation is commonly associated to a reduction in plant fitness, because the reduction in the number of seeds produced. However habitat fragmentation might also affect plant fitness through a reduction in the quality of the seeds produced, as for example in seed viability, seed size or in seed germination. These factors could be affected because small populations size and high degree in isolation, frequently found in fragments, could induce to the loss of genetic population variability and the expression of inbreeding depression. Here I show the effect of forest fragmentation on the quality of seeds produced in Lapageria rosea, quality being measured as seed viability, seed size and seed germination. I collected seeds from the Maulino forest in central Chile, specifically from plants living in forest fragments and continued forest. Seed viability, seed size and seed germination capacity was estimated under laboratory conditions. Results indicated that seeds from forest fragments and from continuous forest have similar percentages of viability, although these percentages are more variable among fruits from forest fragments. Moreover, seeds from forest fragments are smaller than seeds from continuous forest. Finally, seeds from fragments have less germination capacity than seeds from continued forest. These reductions could determinate a negative effect on seedling establishment in fragmented populations and possibly in the long term population survival.
\end{abstract}

Key words: habitat fragmentation, seeds, Lapageria rosea, central Chile.

\section{INTRODUCCIÓN}

La fragmentación del hábitat puede afectar la adecuación biológica de las plantas al reducir la cantidad de semillas producidas, debido a que en poblaciones más pequeñas y aisladas disminuye la cantidad y calidad del polen conespecífico que llega a los estigmas (Aizen \& Feinsinger 1994a, 1994b, Ågren 1996, Morgan 1999, Cunningham 2000a, 2000b). Un aspecto menos 
considerado en la literatura actual es el efecto de la fragmentación del hábitat sobre la calidad de las semillas producidas por las plantas y sus efectos sobre la adecuación biológica.

La fragmentación del hábitat podría inducir a que las plantas generen semillas de menor calidad porque poblaciones pequeñas y aisladas son más propensas a experimentar depresión por endogamia, por aumento en los cruzamientos dentro de la misma planta o entre plantas vecinas usualmente mayormente emparentadas (Jennersten 1988, Barrett \& Kohn 1991, Ellstrand \& Elam 1993, Routley et al. 1999). Dentro de las posibles consecuencias de la depresión por endogamia se encuentra la reducción en la viabilidad, el tamaño o la capacidad germinativa de las semillas producidas (Charlesworth \& Charlesworth 1987, Menges 1991, Heschel \& Paige 1995, Wolf 1995, Ramsey \& Vaughton 1996). Estos atributos de las semillas han sido tradicionalmente asociados a la calidad de la progenie, puesto que su reducción determina usualmente menores probabilidades de reclutamiento de las plántulas (Baskin \& Baskin 1998, Silvertown \& Charlesworth 2001). Por ejemplo, en muchas especies de plantas las semillas más pequeñas generan plántulas de menor tamaño y por lo tanto menos competitivas. Esto puede representar una desventaja en sistemas donde la competencia es importante y por lo tanto podría determinar una reducción en la adecuación biológica (Venable \& Brown 1988, Baskin \& Baskin 1998, Vaughton \& Ramsey 1998, Silvertown \& Charlesworth 2001). Así, en diversas especies se ha encontrado que las semillas provenientes de poblaciones pequeñas y aisladas presentan menor capacidad germinativa que las semillas provenientes de poblaciones de mayor tamaño y menos aisladas (Menges 1991, Heschel \& Paige 1995, Bruna 1999). Sin embargo, en otras especies el tamaño de la población o el grado de aislamiento no afectan la germinación (Morgan 1999, Kéry et al. 2000, Luijten et al. 2000, Mavraganis \& Eckert 2001).

El objetivo de este trabajo es determinar si la fragmentación del bosques maulino en Chile central afecta la calidad de las semillas producidas en la enredadera nativa Lapageria rosea Ruiz et Pav. (Philesiaceae), o "copihue". Esta especie habita en los bosques del centro-sur de Chile y Argentina (Hoffmann 1997) y se encuentra en ambientes sometidos a intensa fragmentación. En fragmentos de bosque la frecuencia de visitas de polinizadores a las flores de L. rosea disminuye, reduciendo el número total de semillas producidas por fruto y por planta (Henríquez 2002). Además, las poblacio- nes de L. rosea evidencian una reducción de la variabilidad genética (Henríquez 2002). Por lo tanto, en fragmentos de bosque las plantas podrían presentar una disminución en la adecuación biológica respecto del bosque continuo, por reducción en la calidad de las semillas producidas. Para evaluar el efecto de la fragmentación sobre la calidad de las semillas producidas, comparé semillas colectadas desde poblaciones de fragmentos y semillas colectadas de un bosque continuo no fragmentado, estimando la viabilidad, tamaño y capacidad germinativa de estas.

\section{MATERIALES Y MÉTODOS}

\section{Características generales de la especie}

Lapageria rosea es autocompatible, sin embargo, debido a que produce un bajo número de flores por individuo, tiene un período de floración extenso y las flores presentan tendencia a la protoginia, experimentando esencialmente reproducción cruzada (Humaña \& Riveros 1994). Sus polinizadores principales son el picaflor Sephanoides sephaniodes y el abejorro Bombus dahlbomii (Humaña \& Riveros 1994). Lapageria rosea produce bayas de 3 a $6 \mathrm{~cm}$ de largo, de color verde-amarillo cuando están maduros. Los frutos producidos en fragmentos de bosque contienen aproximadamente $10 \%$ menos semillas que los frutos producidos en bosque continuo (69 y 82 semillas en promedio, respectivamente; Henríquez 2002).

\section{Área de estudio}

Este estudio fue realizado en el bosque maulino costero de Chile central ( $\left.35^{\circ} 59^{\prime} \mathrm{S}, 72^{\circ} 41^{\prime} \mathrm{O}\right)$. El bosque maulino ha sido sometido a una intensa fragmentación y durante los últimos 25 años ha sido reemplazado por plantaciones de la especie exótica Pinus radiata (CONAFCONAMA-BIRF 1999). Actualmente, gran parte del bosque nativo en esta zona está disgregado en pequeños fragmentos inmersos en una matriz de plantaciones de pino (San Martín \& Donoso 1995, Bustamante \& Castor 1998). La Reserva Nacional Los Queules es una de las pocas áreas protegidas. Contiene 145 ha de bosque nativo protegido, que están inmersas en un área mayor de bosque continuo de 600 ha en total. En esta reserva y en los fragmentos de bosque aledaños, permanecen especies de plantas en peligro de extinción como Gomortega keule (Queule), Pitavia punctata (Pitao) y Berberidopsis corallina (Michay rojo) (Benoit 
1989). Lapageria rosea se encuentra tanto en la Reserva Nacional Los Queules como en los fragmentos de bosque. Estos fragmentos están rodeados por una matriz de plantaciones de $P$. radiata. Las semillas de $L$. rosea fueron colectadas durante dos periodos reproductivos (año 2000 y 2001), desde frutos maduros obtenidos de plantas de la Reserva Nacional Los Queules (bosque continuo) y de plantas de cuatro fragmentos de bosque nativo de distintos tamaños (entre 1 y 6 ha).

\section{Viabilidad de las semillas}

La viabilidad de las semillas fue expresada como el porcentaje de semillas viables por fruto y fue estimada a través de la prueba de Tetrazolium. Esta consiste en exponer embriones disectados a una solución al $0,1 \%$ de 2,3,5triphenyl-2H-tetrazolium chloride (TTC) por 24 h. Este compuesto reacciona con los iones $\mathrm{H}$ liberados por el proceso de respiración de los embriones vivos, causando un cambio de color en los tejidos desde blanco-amarillo a púrpura (Baskin \& Baskin 1998). En este trabajo la viabilidad de las semillas fue expresada como el porcentaje de semillas viables por fruto, extrayendo al azar semillas desde 36 frutos del bosque continuo y 39 frutos de fragmentos (10 semillas de cada fruto y un fruto de cada planta).

\section{Tamaño de las semillas}

El tamaño de las semillas, expresado como biomasa, fue estimado mediante peso seco extrayendo al azar semillas desde 22 frutos (un fruto de cada planta y 10 semillas de cada fruto) colectados de plantas distintas del bosque continuo, y 22 frutos desde plantas distintas de los fragmentos. Las semillas fueron deshidratadas a $100{ }^{\circ} \mathrm{C}$ durante tres días y posteriormente pesadas. En total, se estimó el tamaño a 220 semillas de bosque continuo y 220 semillas de fragmentos.

\section{Capacidad germinativa de las semillas}

La capacidad germinativa de las semillas producidas fue estimada mediante experimentos de laboratorio tipo "jardín común" donde semillas de los dos orígenes, de bosque continuo y de fragmentos, fueron puestas bajo condiciones ambientales similares, con fotoperiodo de $8 \mathrm{~h}$, $20{ }^{\circ} \mathrm{C}$ y $10{ }^{\circ} \mathrm{C}$ de temperatura, y agua por riego ad libitum.

Las semillas fueron colectadas desde 16 plantas del bosque continuo y 10 plantas de los fragmentos de bosque (1 fruto de cada planta).
Se escogieron al azar 10 semillas de cada fruto, las cuales fueron puestas en una cápsula de Petri con papel filtro. Regularmente, cada 7 días, durante 5 meses, se estimó el porcentaje de semillas germinadas por cápsula. Las semillas fueron consideradas como germinadas cuando la radícula emergió al menos $2 \mathrm{~mm}$ desde la cubierta. Los datos fueron analizados mediante un análisis de varianza de medidas repetidas (Sokal \& Rohlf 1995), tomando el lugar de procedencia de las semillas (bosque continuo y fragmentos de bosque) como fuente de variación y considerando el porcentaje de germinación acumulado cada 15 días como la variable dependiente. Los porcentajes de germinación obtenidos fueron transformados mediante transformación arcoseno, para satisfacer los requerimientos de normalidad de los datos del Análisis de Varianza. Las semillas no germinadas se encontraron muertas, según se constató a través de la prueba de Tetrazolium (Baskin \& Baskin 1998).

\section{RESULTADOS}

\section{Viabilidad de las semillas}

En general la viabilidad de las semillas fue alta, tanto en semillas del bosque continuo como en semillas de los fragmentos, siendo la moda 100 $\%$ en ambos casos (Fig. 1). El promedio de viabilidad correspondió a $90 \%$ en las semillas provenientes del bosque y $80 \%$ en las semillas provenientes de los fragmentos. Aunque en términos generales el porcentaje de viabilidad no difirió significativamente entre semillas provenientes del bosque continuo y de los fragmentos (Prueba U de Mann-Whitney; $\mathrm{U}_{38,35}=593$; $\mathrm{P}=0,25)$, la varianza en estos porcentajes fue aproximadamente tres veces mayor en las semillas de los fragmentos que en las semillas del bosque continuo (227 y 670 respectivamente, $\left.\mathrm{F}_{38,35}=2,95 ; \mathrm{P}=0,0015\right)$. De hecho, el porcentaje de viabilidad varió entre un 50 y $100 \%$ en las semillas del bosque continuo, y entre un $0 \mathrm{y}$ $100 \%$ en las semillas de los fragmentos (Fig. 1). En el caso de las semillas de los fragmentos, el $18 \%$ del total de frutos estudiados presentó una viabilidad de semillas inferior al $50 \%$, mientras que en el bosque continuo ningún fruto presentó viabilidad inferior al $50 \%$.

\section{Tamaño de las semillas}

El tamaño de las semillas de los fragmentos fue menor que el tamaño de las semillas del bosque continuo (prueba U de Mann-Whitney; $\mathrm{U}_{219}=$ 

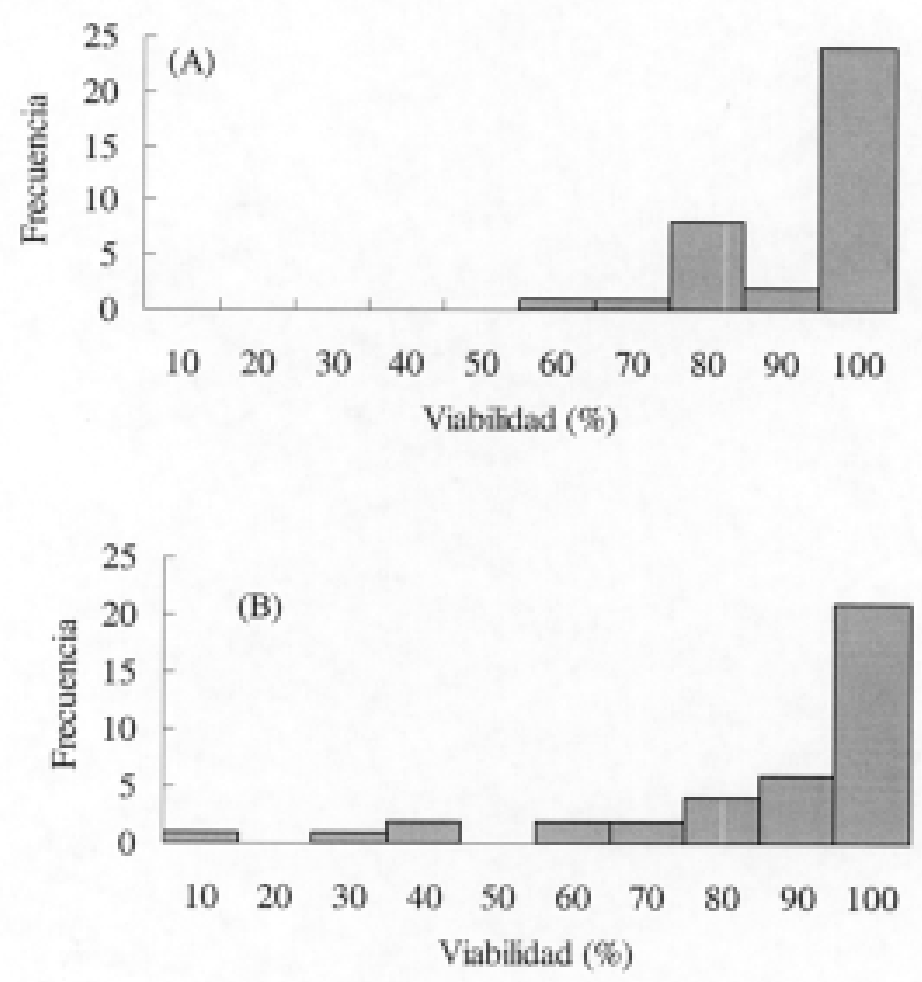

Fig. 1: Distribución de frecuencias de los porcentaje de viabilidad de semillas de Lapageria rosea provenientes de (A) fragmentos de bosque, y (B) bosque continuo.

Frequency distribution of seed viability percentage in Lapageria rosea for seeds collected from (A) forest fragments, and (B) continuous forest.

19.028; $\mathrm{P}<0,001)$. El peso varió entre 10 a 42 $\mathrm{mg}$ en las semillas de los fragmentos y 7 a 39 mg en las semillas del bosque continuo (Fig. 2), siendo las primeras aproximadamente $10 \%$ más pequeñas que las semillas del bosque continuo, $25 \pm 0,5 \mathrm{mg}$ para los fragmentos y $28 \pm 0,5 \mathrm{mg}$ para el bosque continuo (promedio $\pm 1 \mathrm{EE}$ ).

\section{Capacidad germinativa de las semillas}

Las semillas provenientes del bosque continuo presentaron mayores porcentajes de germinación que las semillas de los fragmentos, prácticamente en todos los tiempos medidos. Es decir, se determinó un efecto significativo de la procedencia de las semillas (Tabla 1, Fig. 3). También, estas germinaron más rápidamente que las semillas de los fragmentos. De hecho, las semillas del bosque continuo comenzaron a germinar a los 35 días del experimento, mientras que en los fragmentos se registraron las primeras semillas germinadas solamente a los 60 días. El $50 \%$ de germinación se alcanzó aproximadamente a los 90 días en las semillas provenientes del bos- que continuo, mientras que un porcentaje similar se alcanzó solamente a los 130 días en las semillas provenientes de los fragmentos de bosque. Aproximadamente a los 150 días de experimento las curvas de germinación se estabilizaron, alcanzando un máximo de $77 \%$ para semillas de bosque continuo y $60 \%$ para semillas de fragmentos de bosque. La interacción entre el tiempo y el origen de las semillas también fue significativa (Tabla 1), sin embargo esto se debe a que inicialmente, durante el día 30, no se detectaron diferencias significativas entre semillas de bosque continuo y fragmentos (prueba a posteriori de Tukey; $\mathrm{P}>0,05$ ). Posteriormente a esta fecha, las semillas del bosque continuo presentaron siempre mayores porcentajes de germinación que las semillas de los fragmentos (prueba a posteriori de Tukey; $\mathrm{P}<0,05)$.

\section{DISCUSIÓN}

El reclutamiento de plántulas vía semillas es esencial para la sobrevivencia de las poblacio- 


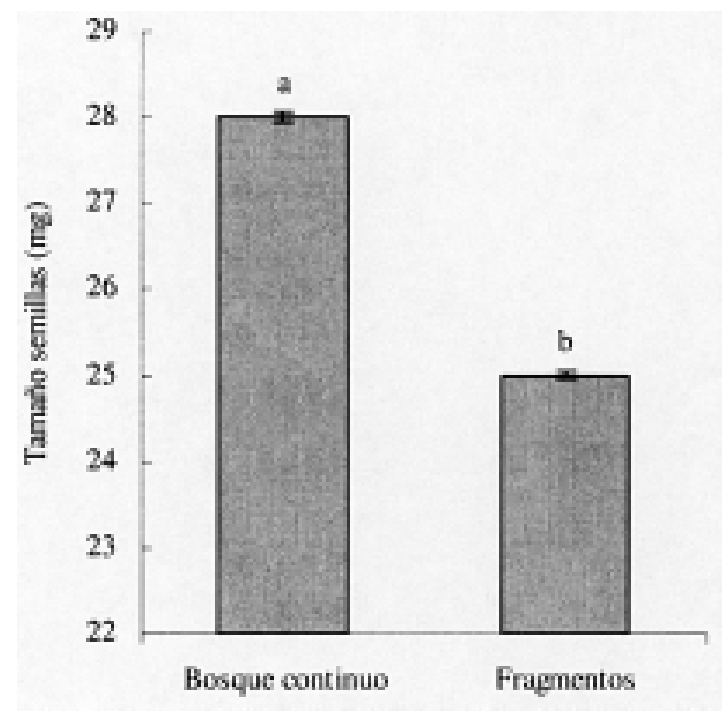

Fig. 2: Tamaño de las semillas (expresado como el promedio del peso de las semillas, en $\mathrm{mg} \pm \mathrm{EE}$ ) de Lapageria rosea provenientes de bosque continuo y fragmentos en el bosque maulino.

Seed size (expressed as the mean seed weight, in $\mathrm{mg} \pm$ $\mathrm{SE}$ ) in Lapageria rosea from continuous forest and forest fragments. nes a largo plazo ya que permite la mantención de la diversidad genética en las poblaciones de plantas, y por lo tanto permite la adaptación a ambientes que cambian (Hamilton 1999). Por lo tanto, aquellos factores que reduzcan el reclutamiento vía semillas, como la disminución en la calidad de estas, pueden ser vistos como amenazas para la sobrevivencia de las poblaciones a largo plazo.

En Lapageria rosea la fragmentación del hábitat afecta la calidad de las semillas producidas. Las semillas provenientes de fragmentos de bosque presentan porcentajes de viabilidad más variables, son de menor tamaño, germinan más lentamente y presentan menores porcentajes de germinación que las semillas provenientes de bosque continuo. Estas características podrían limitar el establecimiento de las plántulas en los fragmentos. De hecho, en bosques tropicales se ha comprobado que la disminución en la germinación de semillas en la especie Heliconia acuminata puede llegar a limitar el reclutamiento de plántulas en la especie, afectando su demografía (Bruna 2002).

El menor tamaño de las semillas producidas en los fragmentos podría reducir el reclutamiento de las plántulas al producir plántulas

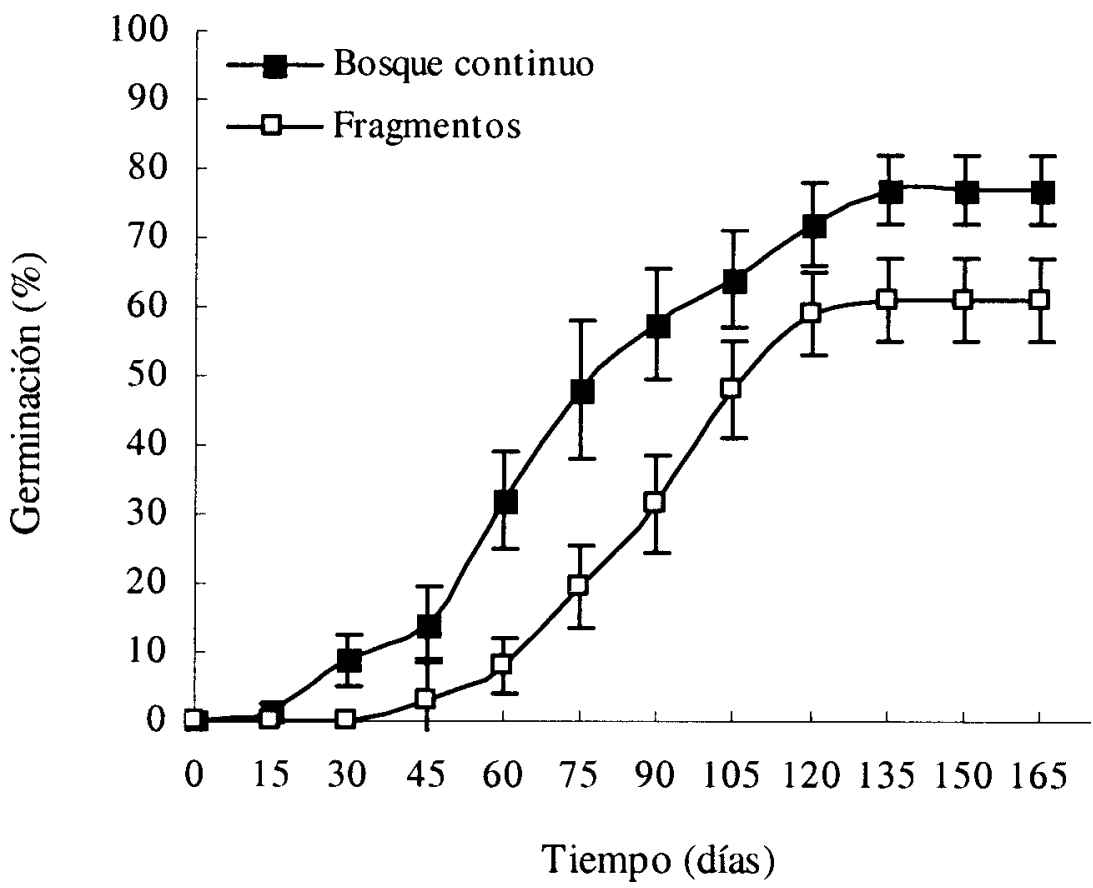

Fig. 3: Germinación de semillas (expresada como el porcentaje de semillas germinadas, promedio \pm $\mathrm{EE}$ ), en semillas provenientes de frutos de Lapageria rosea recolectados desde bosque continuo y fragmentos en el bosque maulino.

Seed germination (expressed as the mean percentage of germinated seed \pm SE) in seeds collected from fruits of Lapageria rosea growing in continuous forest and in forest fragments. 


\section{TABLA 1}

Análisis de varianza de medidas repetidas para el porcentaje de germinación en semillas de Lapageria rosea provenientes de dos orígenes: bosque continuo y fragmentos del bosque maulino. Datos transformados a arcoseno

Repeated messure analysis of variance for seed germination percentage of seeds from Lapageria rosea from two origins: continuous forest and fragments in the Maulino forest. Data were arcsine transformed prior to analysis

\begin{tabular}{lcccc}
\hline Fuente de variación & Grados de libertad & Cuadrado medio & Valor de F & Valor de P \\
\hline Dentro origen & 1 & 9.718 & 4,33 & 0,048 \\
Error & 24 & 2.242 & & $<0,0001$ \\
Entre tiempo & 10 & 14.365 & 94,40 & 0,024 \\
Tiempo x origen & 10 & 322 & 152 & 2,11 \\
Error & 240 & & & \\
\hline
\end{tabular}

de menor tamaño y de menor capacidad competitiva (Baskin \& Baskin 1998, Silvertown \& Charlesworth 2001). Puesto que en los fragmentos de bosque la disponibilidad de agua en el suelo es menor que en el bosque (Henríquez 2002), la producción de semillas más pequeñas podría afectar negativamente el reclutamiento de plántulas ya que estarían sometidas a un ambiente más competitivo (sensu Venable \& Brown 1988). En términos más generales, los bosques de Chile central están asociados a fluctuaciones en los regímenes anuales de precipitaciones, de manera tal que pueden existir años secos, donde las precipitaciones son poco abundantes y tardías, y años húmedos, donde las precipitaciones son abundantes y ocurren tempranamente (Di Castri \& Hajek 1976). Por lo tanto, las semillas producidas en fragmentos podrían presentar aún más desventajas respecto de las semillas producidas en el bosque continuo, particularmente durante años secos. La germinación tardía de las semillas de los fragmentos también representaría una desventaja para el establecimiento de plántulas sobre todo durante los años secos, ya que las plántulas generadas tardíamente tendrían menor capacidad competitiva que las plántulas generadas tempranamente al tener su sistema radicular y su capacidad fotosintética menormente desarrollada.

La mayor variabilidad en los porcentajes de viabilidad en semillas de los fragmentos, y el hecho que en estos aproximadamente el $18 \%$ de los individuos muestreados produzcan semillas con viabilidad inferior al $50 \%$ e incluso esta llegue a ser 0 en uno de los casos, indica que existe una reducción en el tamaño efectivo de las poblaciones en los fragmentos. Es decir, a pesar que algunos individuos producen frutos, la viabi- lidad de las semillas producidas puede ser tan baja, que no estarían contribuyendo en términos genéticos a las generaciones futuras. La reducción en el tamaño efectivo de los fragmentos podría inducir a un incremento en la deriva génica y a una pérdida de la heterocigosidad, y por lo tanto contribuir al deterioro genético futuro de las poblaciones (Hartl \& Clark 1997).

El conjunto de respuestas encontradas en este trabajo, es decir que las semillas de los fragmentos produzcan frutos con semillas no viables o con viabilidad baja, que sean de menor tamaño y que estas presenten reducción en la capacidad de germinación, sugieren que existe depresión por endogamia en las poblaciones de los fragmentos. De hecho, información adicional referente a la menor calidad del polen producido en los fragmentos, a la menor variabilidad genética y a mayores índices de fijación Fis detectados en plántulas de los fragmentos apoyan esta hipótesis (Henríquez 2002).

Por último, cabe destacar la gran gama de respuestas, negativas o neutras, encontradas en la literatura respecto del efecto de la fragmentación del hábitat sobre el desempeño de las semillas en diferentes especies (Menges 1991, Heschel \& Paige 1995, Bruna 1999, 2002, Kéry et al. 2000, Luijten et al. 2000, Mavraganis \& Eckert 2001). Factores fundamentales y que deben ser incorporados al momento de hacer predicciones respecto del desempeño de las semillas son aspectos relacionados con la historia natural de cada especie. En particular la dependencia de factores abióticos para la polinización y cómo estos pueden ser alterados por la fragmentación (Murcia 1996, Aizen 1998) y la longevidad de las plantas. Así, si el fenómeno de fragmentación es reciente y las plantas son longevas podría existir depresión por endoga- 
mia disminuyendo el desempeño de las semillas. Por el contrario, plantas de vida corta y que han estado sometidas a una larga historia de fragmentación, podrían no evidenciar depresión por endogamia debido a que los alelos deletéreos ya habrían sido eliminados. Este aspecto ha sido muy poco estudiado en el contexto de la fragmentación del hábitat.

\section{AGRADECIMIENTOS}

Agradezco a Corporación Nacional Forestal (CONAF) y a la Forestal Millalemu por permitirme trabajar en la Reserva Nacional Los Queules y en los fragmentos de bosque. Además, agradezco a Carlos Valdivia por su ayuda en el campo. Este trabajo fue posible gracias al financiamiento de los proyectos FONDECYT 1981050 y 1010852 otorgados al Dr. J. Simonetti. Por último, doy las gracias a CONICYT por las becas de doctorado y de término de tesis, y al Departamento de Postgrado y Postítulo de la Universidad de Chile, por la Beca Parcial de Tesis de Postgrado.

\section{LITERATURA CITADA}

ÅGREN J (1996) Population size, pollinator limitation, and seed set in the self-incompatible herb Lythrum salicaria. Ecology 77: 1779-1790.

AIZEN MA (1998) Forest fragmentation and plant reproduction: the pollination link. En: Bruns S, S Mantell \& C Tragardh (eds) Recent advances in biotechnology for tree conservation and management: 22-37. IFS, Stockholm, Sweden.

AIZEN MA \& P FEINSINGER (1994a) Habitat fragmentation, native insect pollinators, and feral honey bees in Argentine "Chaco Serrano". Ecological Applications 4: 378-392.

AIZEN MA \& P FEINSINGER (1994b) Forest fragmentation, pollination, and plant reproduction in a Chaco dry forest, Argentina. Ecology 75: 320-341

BARRETT SCH \& JR KOHN (1991) Genetic and evolutionary consequences of small population size in plants: implications for conservation. En: Falk DA \& KE Holsinger (eds) Genetics and conservation of rare plants: 3-30. Oxford University Press, Oxford, United Kingdom. 283 pp.

BASKIN CC \& JM BASKIN (1998) Seeds: ecology, biogeography, and evolution of dormancy and germination. Academic Press, London, United Kingdom. 666 pp.

BENOIT I (ed) (1989) Lista roja de la flora terrestre chilena. Corporación Nacional Forestal, Santiago, Chile. $151 \mathrm{pp}$

BRUNA EM (1999) Seed germination in rainforest fragments. Nature 402: 139.

BUSTAMANTE RO \& C CASTOR (1998) The decline of an endangered temperate ecosystem: the ruil (Nothofagus alessandrii) forest in central Chile. Biodiversity and Conservation 7: 1607-1626.

CHARLESWORTH DR \& B CHARLESWORTH (1987) Inbreeding depression and its evolutionary consequences. Annual Review of Ecology and Systematics 18: 237-268.

CONAF-CONAMA-BIRF (1999) Catastro y evaluación de recursos vegetacionales del bosque nativo de Chile. Informe Regional Séptima Región. Editorial Contempo Gráfica, Santiago, Chile. 118 pp.

CUNNINGHAM SA (2000a) Effects of habitat fragmentation on the reproductive ecology of four plant species in Malle Woodland. Conservation Biology 14: 758-768

CUNNINGHAM SA (2000b) Depressed pollination in habitat fragments causes low fruit set. Proceedings of the Royal Society of London, Biological Sciences 267: 1149-1152.

DI CASTRI F \& E HAJEK (1976) Bioclimatología de Chile. Ediciones Universidad Católica de Chile, Santiago, Chile. 128 pp.

ELLSTRAND NC \& DR ELAM (1993) Population genetic of small population size: implication for plant conservation. Annual Review of Ecology and Systematics 24: 217-242.

HAMILTON MB (1999) Tropical tree gene flow and seed dispersal. Nature 401: 129-130.

HARTL DL \& AG CLARK (1997) Principles of population genetics. Sinauer Associates, Sutherland, Massachusetts, USA. 542 pp.

HESCHEL SM \& KN PAIGE (1995) Inbreeding depression, environmental stress, and population size variation in Scarlet Gilia (Ipomopsis aggregata). Conservation Biology 9: 126-133.

HENRÍQUEZ CA (2002) El dilema de Lapageria rosea en bosques fragmentados: ¿cantidad o calidad de la progenie? Tesis de Doctorado, Facultad de Ciencias, Universidad de Chile, Santiago, Chile. 133 pp.

HOFFMANN AE (1997) Flora silvestre de Chile: zona araucana. Ediciones Fundación Claudio Gay, Santiago, Chile. 258 pp.

HUMAÑA AM \& M RIVEROS (1994) Biología de la reproducción en la especie trepadora Lapageria rosea $\mathrm{R}$. et. P. (Philesiaceae). Gayana Botánica (Chile) 51: 49-55.

JENNERSTEN O (1988) Pollination of Dianthus deltoides (Caryophyllaceae): effects of fragmentation on visitation and seed set. Conservation Biology 2: 359-366.

KÉRY M, D MATTHEIES \& HH SPILLMANN (2000) Reduced fecundity and offspring performance in small populations of the declining grassland plants Primula veris and Gentiana lutea. Journal of Ecology 88: 17-30.

LUIJTEN SH, A DIERICK, J GERARD, B OOSTERMEIJER, LEL RAIJMANN \& HCM DEN NIJS (2000) Population size, genetic variation, and reproductive succes in a rapidly declining, self-incompatible perennial (Arnica montana) in The Netherlands. Conservation Biology 14: 1776-1787.

MAVRAGANIS K \& CG ECKERT (2001) Effects of population size and isolation on reproductive output in Aquilegia canadensis (Ranunculaceae). Oikos 95: 300-310.

MENGES ES (1991) Seed germination percentage increases with population size in a fragmented prairie species. Conservation Biology 5: 158-164.

MORGAN JW (1999) Effects of population size on seed production and germinability in an endangered, fragmented grassland plant. Conservation Biology 13: 266-273.

MURCIA C (1996). Forest fragmentation and the pollination of Neotropical plants. En: Schelhas J \& $\mathrm{R}$ Greenberg (eds) Forest patches in tropical landscapes: 19-36. Island Press, Washington, District of Columbia, USA. 
RAMSEY M \& G VAUGHTON (1996) Inbreeding depression and pollinator availability in a partially self-fertile perennial herb Blandfordia grandiflora (Liliaceae). Oikos 76: 465-474.

ROUTLEY MB, K MAVRAGANIS \& CG ECKERT (1999) Effect of population size on the mating system in a self-compatible, autogamous plant, Aquilegia canadensis (Ranunculaceae). Heredity 82: 518-528.

SAN MARTÍN J \& C DONOSO (1995) Estructura florística e impacto antrópico en el bosque Maulino de Chile. En: Armesto JJ, C Villagrán \& MK Arroyo (eds) Ecología de los bosques nativos de Chile: 153-168. Editorial Universitaria, Santiago, Chile.

SILVERTOWN J \& D CHARLESWORTH (2001) Plant population biology. Blackwell Science, Oxford, United Kingdom. 347 pp.
SOKAL RR \& FJ ROHLF (1995) Biometry. Freeman and Company, New York, New York, USA. 887 pp.

VAUGHTON G \& M RAMSEY (1998) Sources and consequences of seed mass variation in Banksia marginata (Proteaceae). Journal of Ecology 86: 563-573.

VENABLE DL \& JS BROWN (1988) The selective interactions of dispersal, dormancy, and seed size as adaptations for reducing risk in variable environments. American Naturalist 131: 360-384.

WOLF LM (1995) The genetics and ecology of seed size variation in a biennial plant, Hydrophyllum appendiculatum (Hydrophyllaceae). Oecologia 101: 343-352.

Editor Asociado: Julio Gutiérrez

Recibido el 19 de noviembre de 2002; aceptado el 26 de agosto de 2003 